\title{
Effects of hyaluronic acid combined with anti-inflammatory drugs compared with hyaluronic acid alone, in clinical trials and experiments in osteoarthritis: a systematic review and meta-analysis
}

\author{
Thippaporn Euppayo ${ }^{1}$, Veerasak Punyapornwithaya ${ }^{2}$, Siriwadee Chomdej $^{3}$, Siriwan Ongchai ${ }^{4}$ \\ and Korakot Nganvongpanit, ${ }^{1,5^{*}}$ (D)
}

\begin{abstract}
Background: The objectives are to compare the efficacy of intra-articular hyaluronic acid (IA-HA) alone and in combination with anti-inflammatory drugs (IA-HA + AI), corticosteroids (CS) or non-steroidal anti-inflammatory drugs (NSAIDs) in clinical trials and in vivo and in vitro studies of osteoarthritis (OA).

Methods: Data in the BIOSIS, CINAHL, Cochrane Library, EMBASE and Medline databases were collected and analyzed. Random effects models were used to compute the effect size (ES) of the mean difference in pain reduction scores from baseline and the relative risk (RR) of adverse events. The ES of histological scores in vivo and cartilage metabolism in vitro were also calculated. We conducted sensitivity analysis of blinding and intention-totreat (ITT), compared IA-HA combined with CS vs. IA-HA alone in trials, and compared the effects of HA + $\mathrm{Al}$ vs. AI alone in vitro, including anabolic and catabolic gene expression.

Results: Thirteen out of 382 papers were included for data analysis. In clinical trials, the ES of pain reduction scores within the 1st month was $-4.24(-6.19,-2.29)$; 2nd-12th month, $-1.39(-1.95,-0.82)$; and within one year, $-1.63(-2.19,-1.08)$, favoring IA-HA + AI $(P<0.001)$. The ES of RR was $1.08(0.59,1.98)$, and histological scores was $1.38(-0.55,3.31)$. The ES of anabolic gene expression was $1.22(0.18,2.25)$, favoring $\mathrm{HA}$ alone $(P<0.05)$; catabolic gene expression was $0.74(-0.44,1.53)$, favoring $\mathrm{HA}$ alone; and glycosaminoglycans remaining was $-2.45(-5.94,1.03)$.
\end{abstract}

Conclusions: IA-HA + AI had greater efficacy for pain relief than IA-HA alone within a one-year period. However, HA + Al down-regulated the ACAN gene when compared with HA alone in vitro.

Keywords: Anti-inflammatory drugs, Cartilage, Hyaluronic acid, Osteoarthritis, Systematic review

\footnotetext{
* Correspondence: korakot.n@cmu.ac.th

${ }^{1}$ Animal Bone and Joint Research Laboratory, Department of Veterinary

Biosciences and Public Health, Faculty of Veterinary Medicine, Chiang Mai University, Chiang Mai 50100, Thailand

${ }^{5}$ Excellence Center in Veterinary Bioscience, Chiang Mai University, Chiang

Mai 50200, Thailand

Full list of author information is available at the end of the article
} 


\section{Background}

At present, researchers around the world have concluded that osteoarthritis (OA), or degenerative joint disease, a major common joint disease in humans and animals, cannot be cured [1-5]. Although the cartilage cannot return to normal, some medications, such as corticosteroids (CS) and non-steroidal anti-inflammatory drugs (NSAIDs), are useful for relief of pain and inflammation in affected joints due to their inhibition of inflammatory cytokines [6, 7]. Intra-articular (IA) injection of CS (IA-CS) is allowed by the U.S. Food and Drug Administration (FDA) to reduce synovitis and effusion in OA [8], but NSAIDs are prohibited. Corticosteroids have potent anti-inflammatory effects by inhibiting phospholipase $A_{2}$, reducing pain and effusion to a comparatively greater extent than NSAIDs, but prolonged use of CS may result in negative effects and accelerate OA progression. In in vitro studies, triamcinolone acetonide (TA) reduced glycosaminoglycan (GAG) synthesis and increased GAG degradation [9], and dexamethasone (DEX) induced chondrocyte apoptosis through activation of caspases and suppression of the Aktphosphatidylinositol 3'-kinase signaling pathway [10].

Hyaluronic acid (HA) is a symptomatic slow-acting drug for osteoarthritis (SYSADOA) which is modestly effective in the treatment of moderate knee OA pain [11]. Since 1997, intra-articular injection of HA (IA-HA) in various preparations has been approved by the FDA for knee OA [12]. Previous studies have conducted a meta-analysis of published reports on the use of IA-HA in OA. In nearly all of the papers, HA had a greater effect on pain relief than a placebo [13-20]. Many recent reports have investigated the synergistic effects, drug interactions and decreased cytotoxicity of HA when combined with other drugs, in order to develop more effective OA treatments [21-27].

Much research has been conducted on HA combined with anti-inflammatory drugs (IA-HA + AI), via clinical trials and in vivo and in vitro studies. Some experiments have focused on the synergistic or antagonistic effects of these drug combinations. However, there is a lack of strong evidence in the literature supporting the efficacy of IA-HA + AI. This has led to the objective of this study: to analyze the effects of IA-HA + AI in clinical trials and in vivo and in vitro studies, using a systematic review and meta-analysis, to gain new insights that may help clinicians treat OA with IA-HA + AI with more confidence.

\section{Methods}

\section{Literature search}

Literature in the BIOSIS, CINAHL, Cochrane Library, EMBASE and Medline databases was included in a computerized search. We selected studies published in
English that were performed from 1980 to 2016, using the following keywords: articular cartilage, chondrotoxicity, corticosteroid, degradation, hyaluronic acid, and NSAIDs. Reviewers screened titles, evaluated the eligibility of studies, and contacted the primary authors of abstracts with incomplete data. The results were compared and discussed to resolve any disagreements among the five reviewers (E.T., P.V., C.S., O.S. and N.K.).

\section{Selection}

This study was categorized into three different parts: clinical trials, and in vivo and in vitro studies. Clinical trials consisted of randomized controlled trials that compared IA-HA and IA-HA + AI (CS or NSAIDs). This included IA-HA + AI administered under different conditions: i) injected together; ii) pre-injection with HA; or iii) postinjection with HA. The therapeutic effects of the drugs on OA joints in humans were assessed. In vivo and in vitro studies were those involving: i) normal cartilage; ii) OAinduced models; and iii) spontaneous OA in animals, focusing on the effects of drugs. Chondrocytes and cartilage with or without chemical- or cytokine-induced pathology were included. The positive or negative results of drugs in clinical trials and research experiments were subjected to data analysis. Duplicate articles, studies on systemic effects of drugs, review articles and case reports were excluded. To reduce bias, articles supporting commercial products were also excluded.

\section{Outcome measures}

The outcome measures were different, depending on study type. For clinical trials, the primary outcome was pain relief from drugs recommended for OA [28]. The secondary outcome was adverse events (AE) that can occur after IA injection of drugs for OA [29]. All definitions of AE specified by the authors - such as pain, swelling, redness, heat, or loss of joint function typically related to IA drugs - were measured. For in vivo studies, data on histological scores were compared and analyzed. For in vitro studies, anabolic gene expression, i.e. aggrecan $(A C A N)$ and collagen type II alpha 1 (COL2A1), catabolic gene expression, i.e. ADAM metallopeptidase with thrombospondin type 1 motif 5 (ADAMTS5), cyclooxygenase-2 $(C O X-2)$, interleukin 1 beta $(I L-1 \beta)$, matrix metalloproteinase-2 (MMP2), matrix metalloproteinase-3 (MMP3), and matrix metalloproteinase-13 (MMP13), and glycosaminoglycans (GAG) in chondrocyte pellets or cartilage explants were measured. For in vivo and in vitro experiments using various doses of HA or anti-inflammatory drugs, the highest dose in each study was used to calculate the effect size in order to avoid experimental bias.

\section{Data extraction}

For the three study categories - clinical trials, and in vivo and in vitro studies - data on authors, study 
duration, mean outcome value, year of publication, and sample size were collected and recorded. Mean and standard deviation (SD) of data in graphs was estimated using the WebPlotDigitizer program. For clinical trials, we calculated the pain reduction from baseline of painrelated outcomes at all time points of each trial. We also generated new SD from the data on mean pain reduction scores from baseline over a one-year period (for all time points within one year, minus baseline). Moreover, we analyzed the data on mean pain reduction from baseline by groups: within the 1st month (all time points within the 1st month, minus baseline); and from the 2nd month to the 12th month (all time points during 2nd12th month, minus baseline). We also analyzed the relative risk (RR) of $\mathrm{AE}$ after using IA-HA + AI compared with IA-HA alone. The data for each part of the analysis were pooled using random effects models. The effect size (ES) was calculated at a $P$ value of less than 0.05 and reported as a $95 \%$ confidence interval $(95 \% \mathrm{CI})$. The data were displayed in forest plots to illustrate the values for each part. The heterogeneity of data was presented as a percentage, based on the $I^{2}$ statistic. In this study, R version 3.2.3 was used for analysis of all data.

\section{Sensitivity analysis}

Sensitivity analysis was performed for clinical trials and in vitro studies, but in vivo studies could not be analyzed because of different outcome measurements. Clinical trials were subgrouped into trials that reported blinding (single-blinding and double-blinding) and intention-totreat (ITT) analysis (explicitly or not explicitly reported). Meta-regression analysis was used to assess the blinding of randomization and ITT. We directly compared the effects of IA-HA + CS with IA-HA alone. For in vitro studies, we separately analyzed the expression of the anabolic genes $A C A N$ and COL2A1. Moreover, we evaluated the effects of HA + AI compared with each antiinflammatory drug alone on anabolic gene expression, catabolic gene expression, and GAG remaining in chondrocyte pellets or cartilage explants.

\section{Results}

\section{Trials and studies}

The research that was related to our scope included 382 papers. Duplicate papers (both duplicated in various databases or with the same or similar contents) were excluded $(n=69)$. Papers that did not fit the inclusion criteria $(n=249)$ were also excluded. Finally, the following 51 papers were excluded for reasons of: (1) not using HA as a control (7 papers); (2) studies of HA compared with anti-inflammatory drugs (41 papers); and (3) insufficient data for pooling (3 papers). Ultimately, 13 papers were found to be eligible and corresponded to inclusion criteria (Fig. 1). Twelve studies were published as original articles and one was published as a preliminary study.

Part I, clinical trials - Five trials [30-34] related to knee OA were included, with duration ranging from one week to one year. The average age range of patients was 40-80 years.

Part II, in vivo studies - Research related to the topic included three papers: in a rabbit OA model, studies on HA combined with liposomal celecoxib (CLX) [35], and HA combined with cortisone [36]; and in a rat OA model, cross-linked hyaluronic acid hydrogel (cHA gel) combined with DEX in surgery-induced OA [37].

Part III, in vitro studies - There were five studies [22, $23,38-40]$, including anabolic gene (ACAN and COL2A1) expression, catabolic gene (ADAMTS5, COX-2, IL-1 $\beta$, $M M P 2, M M P 3$, and $M M P 13)$ expression, and GAG remaining in chondrocyte pellets or cartilage explants.

The details of each study are shown in Table 1 for clinical trials, and in Tables 2 and 3 for in vivo and in vitro studies.

\section{Clinical trials \\ Risk of bias in clinical trials}

For clinical trials, we reviewed the risk of bias in five randomized trials. Two trials reported double-blinding $[30,34]$, two trials reported single-blinding [32, 33], and the other gave no details [31]. Three trials reported ITT analysis [30, 32, 34].

\section{Comparison between treatments}

For clinical trials, mean differences in pain reduction scores - based on the visual analog scale (VAS) and the Western Ontario and McMaster Universities Arthritis Index (WOMAC) pain score - within the 1st month, from the 2nd to 12th month, and within one year after injection were recorded in forest plots, as shown in Fig. 2a-c, respectively. In Fig. 2a, the effect size (ES) of the random effects model for the mean difference in pain reduction score within the 1st month was -4.24 (95\% CI: -6.19 to -2.29 , favoring IA-HA $+\mathrm{AI}$; $P<0.0001)$. The $I^{2}$ value was $95.25 \%$, indicating a substantial amount of heterogeneity. The ES of the random effects model for the mean difference in pain reduction score from the 2nd to 12th month was -1.39 (95\% CI: -1.95 to $-0.82 ; P<0.0001)$. The $I^{2}$ value was $81.43 \%$ and the $P$ value of heterogeneity was 0.0001 , favoring IAHA + AI (Fig. 2b). The ES of the random effects model for the mean difference in pain reduction score within one year was -1.63 (95\% CI: -2.19 to -1.08 , favoring IAHA + AI; $P<0.0001)$. The $I^{2}$ value was $83.48 \%$, with $P$ value $<0.0001$ for the heterogeneity test (Fig. 2c). These results indicated that, in the first month after injection, using IA-HA + AI significantly reduced pain scores compared with IA-HA alone, by 4.24 -fold $(P<0.0001)$. 


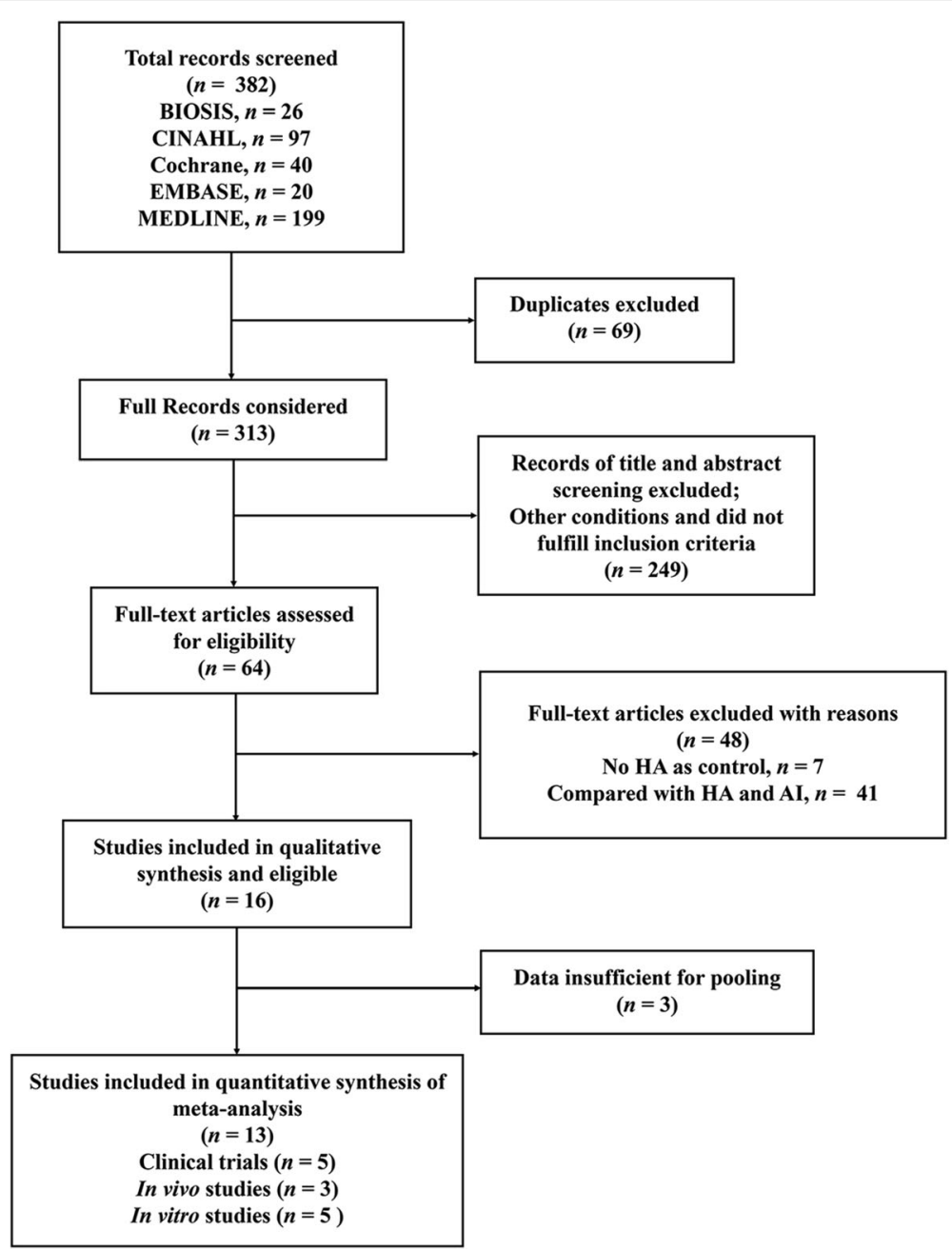

Fig. 1 Flow diagram of articles to evaluate the systematic review and meta-analysis. Al, anti-inflammatory drugs; HA, hyaluronic acid; $n$, number of extracted papers

During the 2nd to 12th month, using IA-HA + AI significantly reduced pain scores compared with IA-HA alone, by 1.39 -fold $(P<0.0001)$. Over a one-year period, using IA-HA + AI significantly reduced pain scores compared with IA-HA alone, by 1.63 -fold $(P<0.0001)$.

\section{Safety of injection}

The RR of AE using IA-HA + AI compared with IA-HA alone in clinical trials is shown in Fig. 3. The ES of the random effects model in terms of RR was 1.08 (95\% CI: 0.59 to $1.98 ; P=0.80)$. The $I^{2}$ value was $0 \%$ and the $P$ value of heterogeneity was 0.29 . These results suggest that using IA-HA + AI was a related factor in RR, causing $8 \%$ more (1.08-fold) AE than IA-HA alone; however, this was not a significant difference.

\section{In vivo studies}

Mean differences in histological scores are displayed in a forest plot in Fig. 4. The ES of the random effects model was 1.38 (95\% CI: -0.55 to $3.31 ; P=0.16)$ and the $I^{2}$ value was $87.35 \%$, favoring $\mathrm{HA}$. There was no significant difference between using IA-HA + AI and IA-HA alone when considering the severity of histological scores in OA animal models $(P<0.05)$.

\section{In vitro studies}

Meta-analysis of cartilage structure synthesis and degradation is exhibited in Fig. 5. For anabolic gene expression ( $A C A N$ and COL2A1) (Fig. 5a), the ES of the random effects model was 1.22 (95\% CI: 0.18 to 2.25 , favoring $\mathrm{HA}$ alone; $P=0.0211)$. The $I^{2}$ value was $51.71 \%$ and the $P$ value of heterogeneity was 0.038 . The ES of catabolic gene expression (ADAMTS5, COX-2, IL-1 $\beta$, $M M P 2, M M P 3$, and $M M P 13)$ was $0.74(-0.04,1.53)$, favoring HA alone $(P=0.0616)$ (Fig. $5 \mathrm{~b}) ; I^{2}$ value was $30.99 \%$ ( $P$ value of heterogeneity $=0.1992)$. For GAG in chondrocyte pellets or cartilage explants (Fig. 5c), the ES of the random effects model was -2.45 (95\% CI: -5.94 


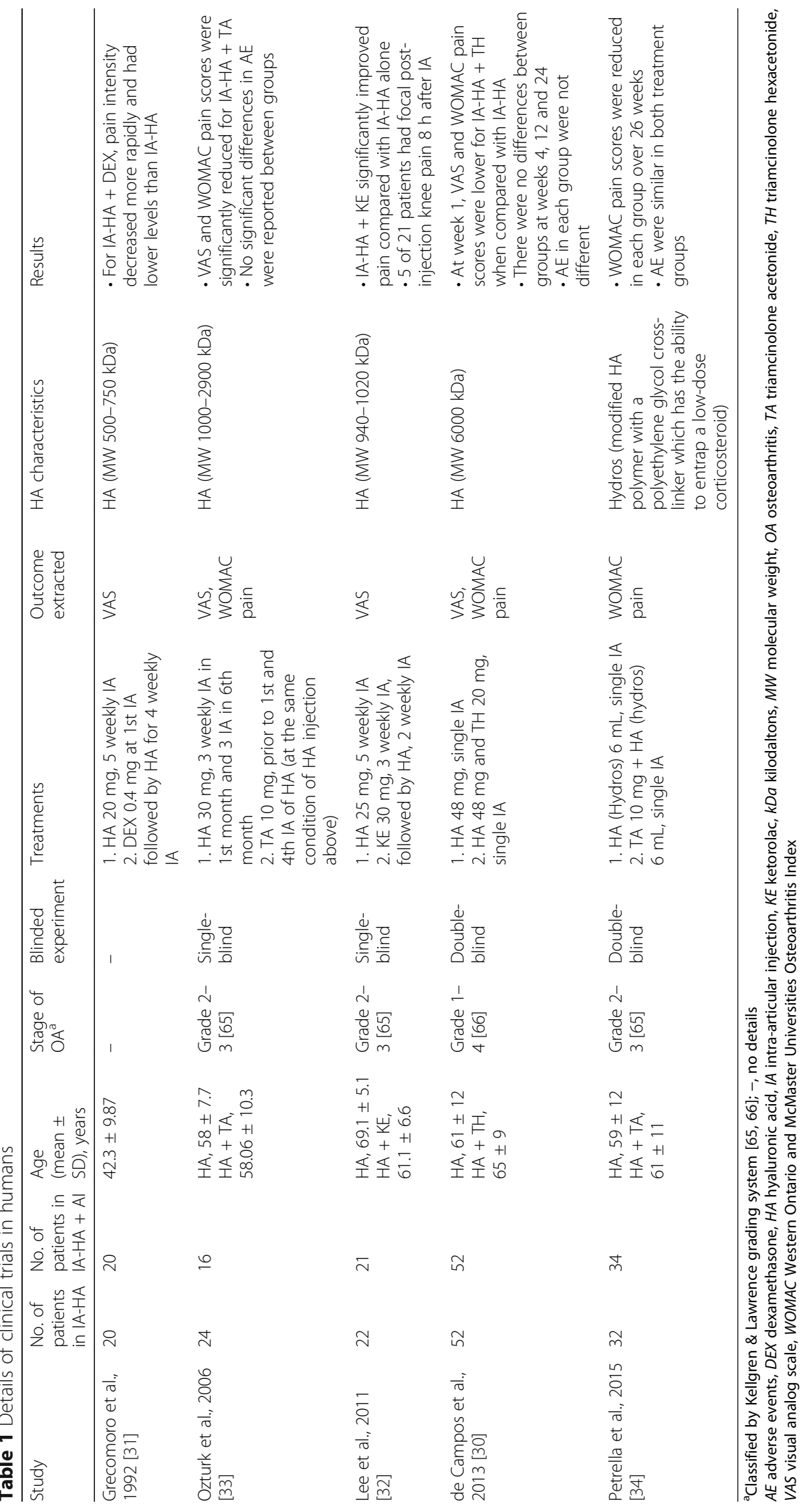




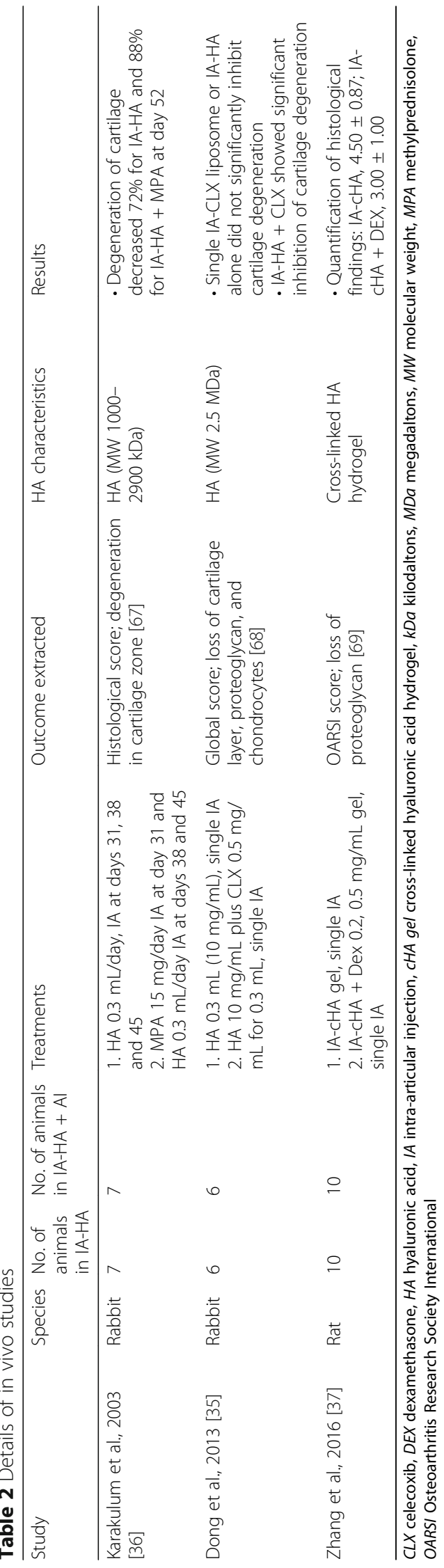




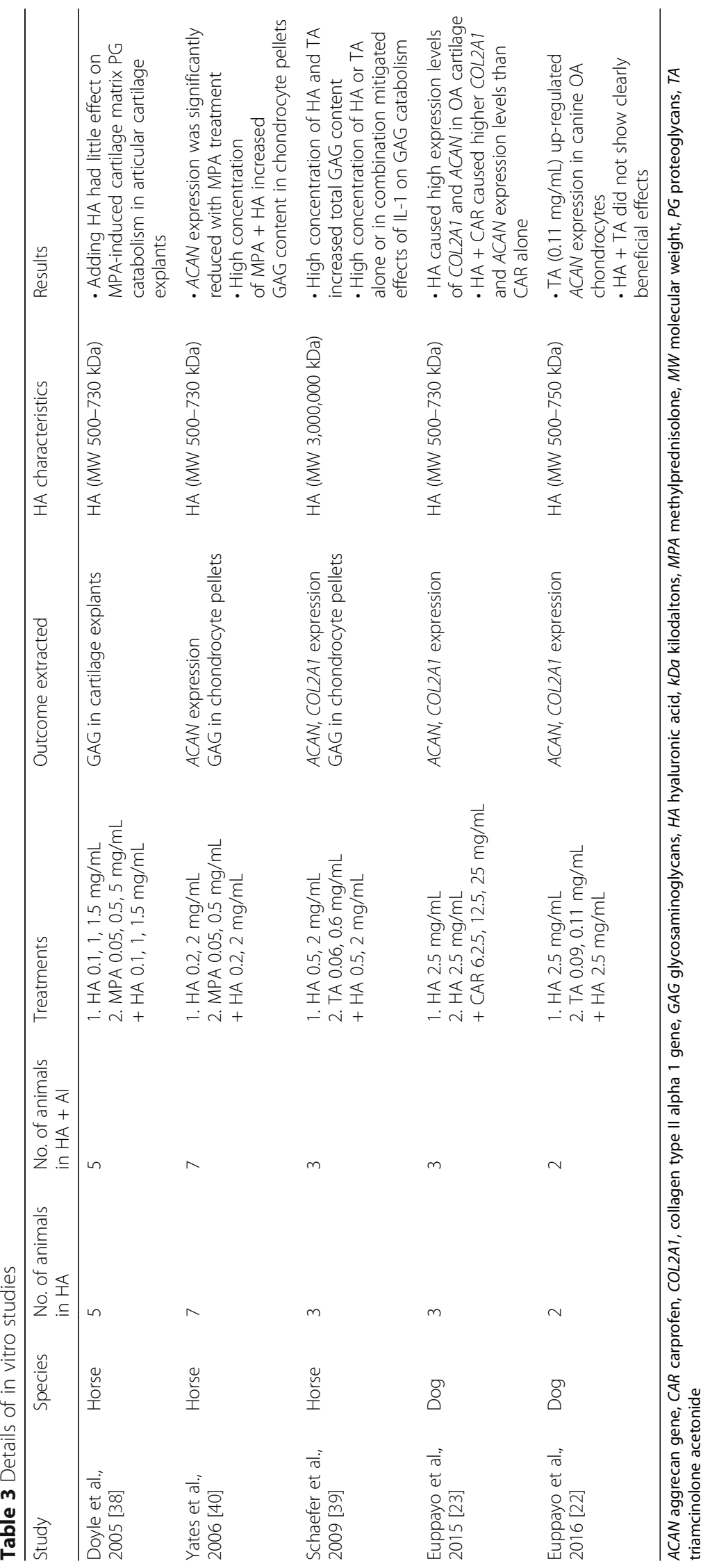




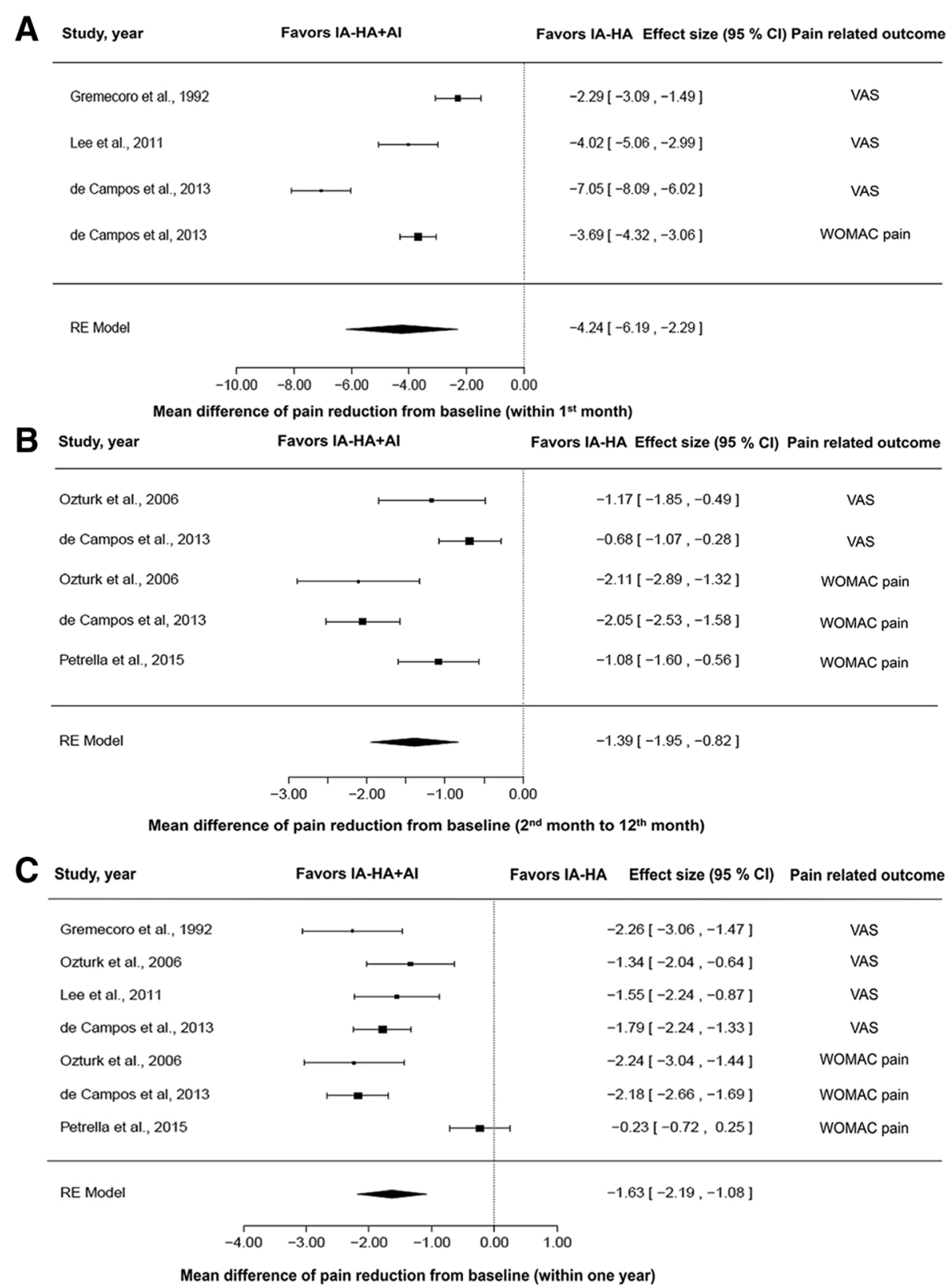

Fig. 2 Forest plots of pain-related outcomes in clinical trials: mean difference in pain reduction from baseline within the 1st month (a), 2nd month to 12th month (b), and within one year (c). Al, anti-inflammatory drugs; Cl, confidence interval; HA, hyaluronic acid; IA, intra-articular; RE, random effects model; VAS, visual analog scale; WOMAC, Western Ontario and McMaster Universities Osteoarthritis Index

to $1.03 ; P=0.1678)$. The value of $I^{2}$ was $91.24 \%$ at $P=0.0019$. These results suggested that $\mathrm{HA}+\mathrm{AI}$ downregulated anabolic gene expression when compared with HA alone $(P<0.05)$. HA alone induced catabolic gene expression more than HA + AI, but there was no significant difference. For GAG remaining in chondrocyte pellets or cartilage explants, there was no significant difference between HA + AI and HA alone.

\section{Sensitivity analysis}

For clinical trials, we first confined pooled analysis to three trials that reported ITT analysis [30, 32, 34]. In this subset, the ES was -1.18 (95\% CI: -2.15 to -0.22 , favoring IA-HA + AI; $P=0.0165)$. The pooled analysis of trials that reported single-blind or double-blind methodology included four trials [30, 32-34]. The ES of this subset was -1.22 (95\% CI: -1.93 to -0.51 , favoring 


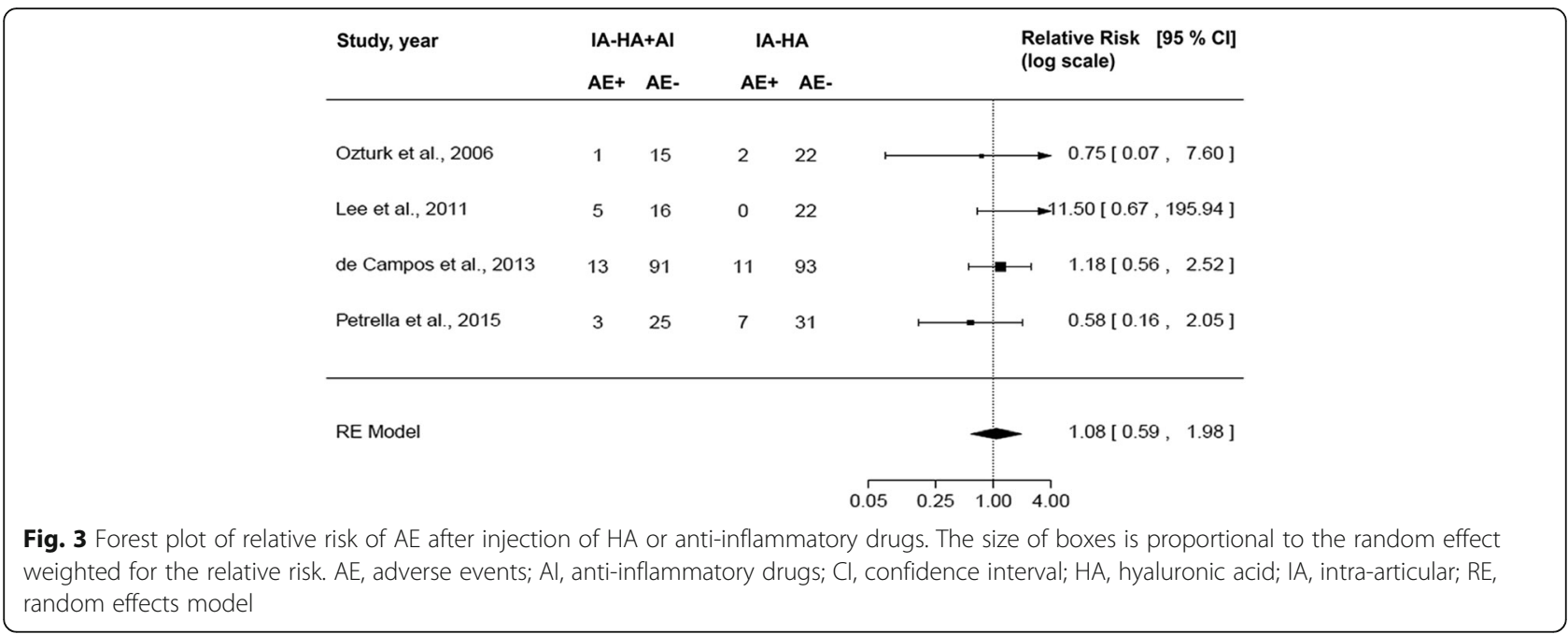

IA-HA + AI; $P=0.0008)$. Meta-regression analysis identified no significant difference between groups of blinding or ITT $(P<0.05)$. For the subgroup of IA-HA combined with CS vs. IA-HA alone, the ES was -1.38 (95\% CI: -2.24 to -0.52 , favoring IA-HA + CS; $P=0.0017)$. These results suggested that although we separately analyzed trials which reported only ITT or single/ double-blinding, the results showed the same trends when interpreted for all five papers on clinical trials.

For in vitro studies, when comparing the effect of $\mathrm{HA}+\mathrm{AI}$ vs. HA alone on $A C A N$ expression, the ES was 1.67 (95\% CI: 0.37 to 2.97, favoring HA alone; $P=0.0118$ ); for COL $2 A 1$ expression, the ES was 0.59 (95\% CI: -1.09 to $2.27 ; P=0.49$ ). This meta-analysis showed that HA + AI had no clear effect on COL2A1 expression when compared with HA alone. But when considering anabolic gene expression (including both $A C A N$ and $C O L 2 A 1)$ or in analyzing the subset of the $A C A N$ gene alone, the results showed that $\mathrm{HA}+\mathrm{AI}$ could reduce anabolic gene expression, especially the $A C A N$ gene.

For the effect of HA + AI compared with AI alone on anabolic gene expression levels, the ES was -0.29 (95\% CI: -1.04 to $0.45 ; P=0.439)$. The ES of catabolic gene expression was 0.43 (95\% CI: -0.21 to $1.07 ; P=0.1897$ ), while for GAG remaining in chondrocyte pellets or cartilage explants the ES was -2.6 (95\% CI: -6.61 to 1.41 ; $P=0.2035)$. There was no significant difference between $\mathrm{HA}+\mathrm{AI}$ and AI alone on anabolic or catabolic gene expression levels, or on GAG remaining in chondrocyte pellets or cartilage explants.

\section{Discussion}

For clinical trials, the results of five papers [30-34] showed the same trend in pain relief. Intra-articular injection of NSAIDs (IA-NSAIDs) or IA-CS could act as rapid-onset pain relief - but not long (slow) acting as in the case of IA-HA - as observed from VAS or WOMAC

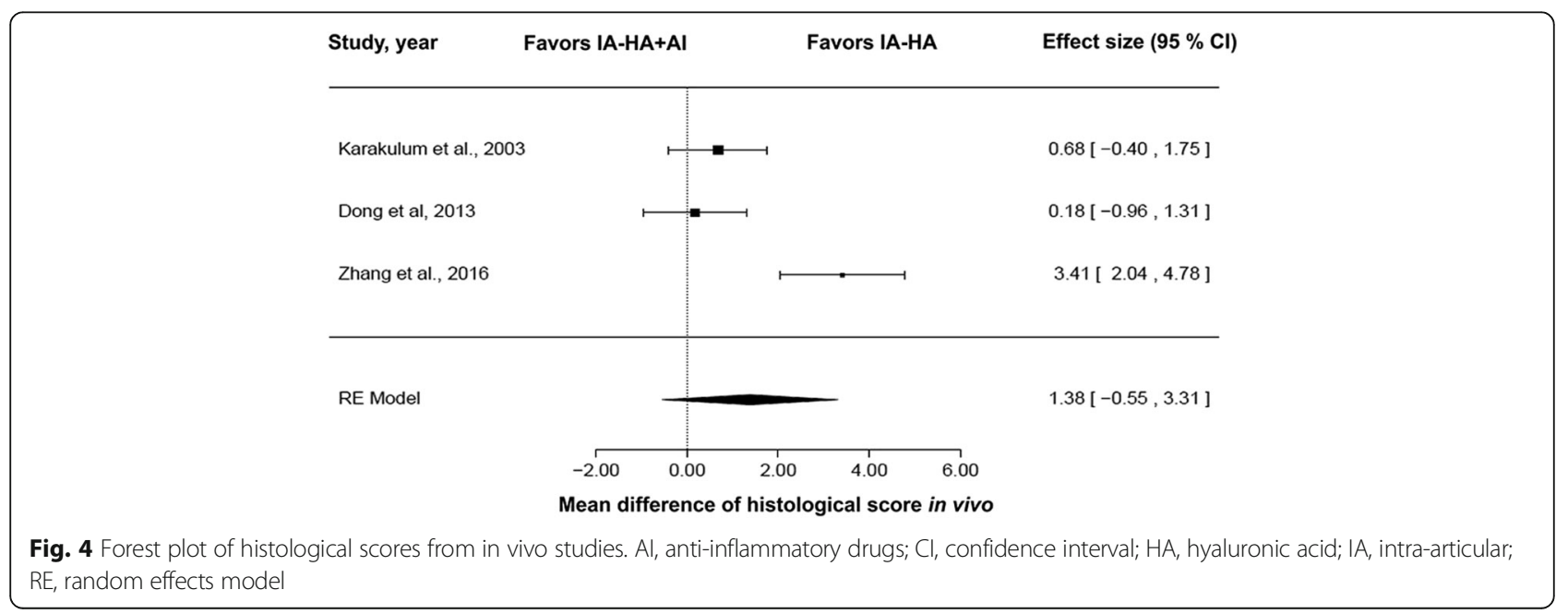




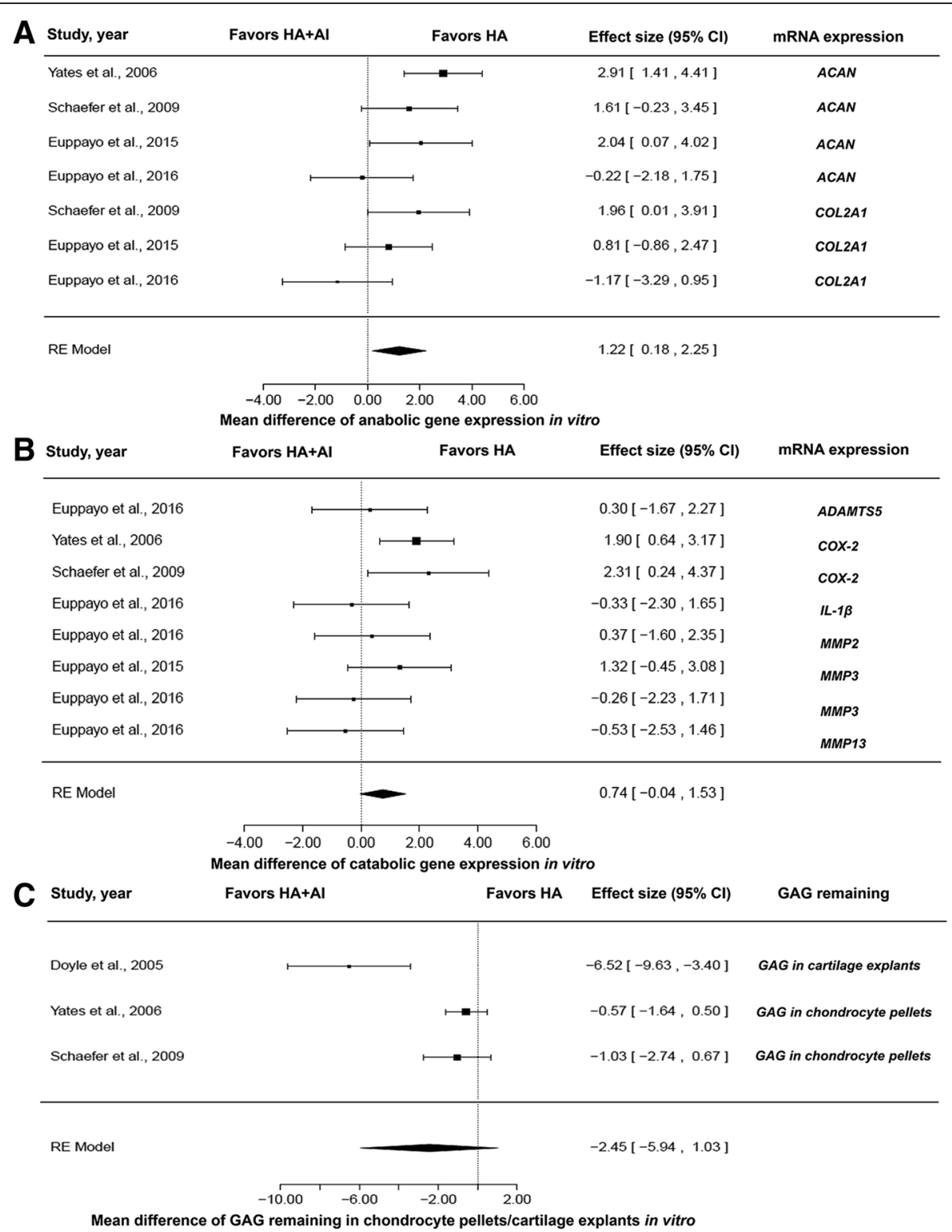

Fig. 5 Forest plots of cartilage structure synthesis and degradation in vitro: (a) ACAN and COL2A1 anabolic gene expression in chondrocytes or cartilage explants, comparing HA + Al and HA; (b) ADAMTS5, COX-2, IL-1B, MMP2, MMP3, and MMP13 catabolic gene expression in chondrocytes or cartilage explants, comparing $\mathrm{HA}+\mathrm{Al}$ and $\mathrm{HA}$; (c) GAG remaining in chondrocyte pellets or cartilage explants, comparing $\mathrm{HA}+\mathrm{Al}$ and $\mathrm{HA}$. ACAN, aggrecan gene; Al, anti-inflammatory drugs; Cl, confidence interval; COL2A1, collagen type II alpha 1 gene; GAG, glycosaminoglycans; HA, hyaluronic acid; RE, random effects model

pain scores within one year, especially at the earliest stage. High molecular weight HA $(6000 \mathrm{kDa})$ was suggested for chronic knee OA pain because it relieved pain by week 12, while triamcinolone hexacetonide could relieve pain within 1 to 2 weeks [41]. Hence, using a combination of IA-HA + AI can be more effective for both short- and long-term pain reduction. This can be used either by pre-treating with anti-inflammatory drugs (IADEX or IA-TA) before a series of HA injections, or by administering in combination at the same time, e.g. IA- ketorolac (KE) combined with HA, or IA-TA combined with Hydros (modified HA polymer with a polyethylene glycol cross-linker), which can be entrapped by TA in its structure.

The reasons for collecting papers based on VAS and WOMAC pain scores, which were used as the main parameters for clinical trials, is because pain intensities were estimated by the patients themselves. The VAS, a unidimensional scale for measuring pain intensity, is the most frequently used pain rating scale [42] and is 
effective for determining average OA pain [43]; while the WOMAC pain subscale was included because it is widely used for measuring OA symptoms and physical disability status [44-47]. Other parameters, such as joint function, stiffness, and joint swelling, are also important in estimating clinical OA, but were not included in this study because of the limited amount of available data for pooling. Moreover, these parameters have no standard scoring system and are more subjective than the WOMAC pain score, so they should not be used for comparison between studies.

Although IA-AI and IA-HA have been widely used for treatment of OA joints, they could cause $\mathrm{AE}$ after injection. Overall RR results indicated that adding CS or NSAIDs in combination with IA-HA did not significantly increase AE compared with IA-HA alone. However, four of the studies included in this paper reported $\mathrm{AE}$, such as severe pain and joint effusion [30, 32-34]; in these studies, the overall percentage of $\mathrm{AE}$ was $10.64 \%$ in the IA-HA group (20 of 188 patients) and $13.02 \%$ in the IA-HA + AI group (22 of 169 patients). It is possible that both IA-HA and IA-HA + AI can cause AE. However, adding AI in the same injection with HA or injecting the drugs before injection of HA did not increase $\mathrm{AE}$ in knee $\mathrm{OA}$ patients. Along with certifying the safety of IA-HA and IA-AI, the European Society for Clinical and Economic Aspects of Osteoporosis and Osteoarthritis (ESCEO) has recommended using IA-HA or IA-CS in symptomatic OA patients [48]. However, the Osteoarthritis Research Society International (OARSI) remains uncertain about recommending the use of IA-HA (except possibly for knee OA, as determined by physician/patient interaction), and has designated it as not appropriate for use in multiple-joint OA [49]. For administration of HA alone, for US-approved HA products for knee OA there was no significant difference in safety outcome and serious AE risk between IA-HA and a saline control [17]. However, AE of IA-HA included: mild transient local reaction; severe postinflammation reactions which could occur due to administration of highly cross-linked high molecular weight HA [50]; immunogenic response [51]; or possibly a crystal-like response to large particles of HA [52]. For administration of IA-CS, infection, post-injection flare, crystal-induced synovitis, cutaneous atrophy and steroid arthropathy were noted as complications [52]. Moreover, steroid-induced (Charcot-like) arthropathy may occur after multiple injections [52].

In this study, we collected papers on HA combined with various types of AI that had different actions of anti-inflammation and analgesia. In step 2 of advanced pharmacological OA management, IA CS could be injected if a patient still had symptomatic OA [53]. A study comparing the effects of IA CS reported that $\mathrm{TH}$ was more effective than MPA on pain reduction at week 3 , but that MPA resulted in a greater decrease in VAS and Lequesne index scores at week 8 of treatment [54]. $\mathrm{TH}$ was found to have a longer duration of action than TA for improvement of weight-bearing joints [55], while DEX produced analgesia similar to morphine in chronic arthritis [56]. Along with IA NSAIDs, there are a few of the previous study when compared with IA CS. KE is a classical NSAID that could be safely used for IA administration in post-operative pain relief [57, 58]. Some NSAIDs also had beneficial effects in in vivo and in vitro studies, e.g. IA CLX could suppress IL-1 $\beta$, TNF, and MMP-3, and improved pathological changes of cartilage, similar to IA HA in a rabbit OA model [59]. Likewise, carprofen could decrease the severity of OA cartilage lesions in conjunction with decreasing the width of osteophytes in dog OA model [60]. Based on this study, IA NSAIDs may be used for clinical treatment of OA.

Degradation of aggrecan and collagen in cartilage structure is a manifestation of OA [53, 54]. When considering drug combinations in experiments, this research revealed that adding IA-CS or IA-NSAIDs in combination with HA may reduce the anabolic effect of HA on cartilage by down-regulating anabolic gene $(A C A N)$ expression when compared with IA-HA alone. Hence, those using IA$\mathrm{HA}+\mathrm{AI}$ should be aware that AI may reduce anabolic gene expression levels. But these combinations had no clear effect on the level of catabolic gene expression and of GAG protein remaining in cartilage, or on histological changes $[61,62]$. This may be due to the use of various histological grading criteria, different drug dosages and study durations, and a limited number of research reports. Further research should be undertaken to confirm these points.

In this study, we analyzed various MW of exogenous HA preparations, including low (MW 500-730 kDa), intermediate (MW 800-2000 kDa) and high MW (average: $6000 \mathrm{kDa}$ ) [63] and newly modified structures of HA to entrap other drugs. The effect of each MW of IA$\mathrm{HA}+\mathrm{AI}$ on OA joints should be collected, but there were a low number of papers for pooling data. Although we included combinations of HA with several different drugs, the results showed significantly reduced pain for the combinations compared with HA alone.

Limitations of this study are a low number of related research reports and small pooled sample size because this research was conducted based on a literature database search [64]. Moreover, in clinical trials of OA, the comparison of IA-AI and IA-HA + AI is interesting to include, but no conclusions can be drawn because of the low number of experiments using anti-inflammatory drugs alone to treat OA for an extended period. However, the strengths of this paper are revealing data on the use of HA plus AI in the field of clinical, in vivo and in 
vitro. To update the new knowledge of $\mathrm{HA}+\mathrm{AI}$ based on increased population of humans and animals, using statistical analysis.

\section{Conclusions}

Published data indicate with a good level of evidence that intra-articular injection of HA combined with antiinflammatory drugs can potentially relieve pain in OA knee patients without increasing serious AE when combined with HA alone. However, in vitro studies indicate caution when using these in combination due to the potential reduction in expression levels of anabolic genes, especially $A C A N$ expression which may encode aggrecan structure in cartilage.

\section{Abbreviations \\ ACAN: Aggrecan gene; AE: Adverse events; CAR: Carprofen; CHA gel: Cross- linked hyaluronic acid hydrogel; Cl: Confident interval; CLX: Celecoxib; COL2A1: Collagen type II alpha 1 gene; CS: Corticosteroids; \\ DEX: Dexamethasone; ESCEO: European Society for Clinical and Economic Aspects of Osteoporosis and Osteoarthritis; GAG: Glycosaminoglycan; HA: Hyaluronic acid; IA: Intra-articular injection; ITT: Intent-to-treat; kDa: Kilodaltons; KE: Ketorolac; MDa: Megadaltons; MPA: Methylprednisolone; MW: Molecular weight; NSAIDs: Non-steroidal anti-inflammatory drugs; OA: Osteoarthritis; OARSI: Osteoarthritis Research Society International; PG: Proteoglycans; RE: Random effect model; RR: Relative risk; SD: Standard deviation; TA: Triamcinolone acetonide; TH: Triamcinolone hexacetonide; VAS: Visual analog scale; WOMAC: Western Ontario and McMaster Universities Osteoarthritis Index}

\section{Acknowledgements}

This research was kindly supported by the Excellence Center in Veterinary Bioscience and Excellence Center in Osteology Research and Training Center, Chiang Mai University, Chiang Mai, Thailand.

\section{Funding}

This study was supported by grants from Excellence Center in Veterinary Bioscience and Excellence Center in Osteology Research and Training Center, Chiang Mai University, Chiang Mai 50,200, Thailand.

\section{Availability of data and materials}

The datasets used and/or analysed during the current study are available from the corresponding author on reasonable request.

\section{Authors' contributions}

TE and KN contributed to the concept of this review. TE, VP, SC, SO and KN reviewed trials and experiments. TE analyzed data and drafted manuscript. VP revised statistical data analysis. KN revised manuscript. All author read, discussed results and approved final manuscript. All authors have read and approved of the final manuscript.

\section{Ethics approval and consent to participate}

It is not applicable, retrospective study and meta-analysis did not require approval by the Animal/Human Ethics Committee, Faculty of Veterinary Medicine, Chiang Mai University.

\section{Consent for publication}

Not applicable.

\section{Competing interests}

The authors declare that they have no competing interests regarding the publication of this paper.

\section{Publisher's Note}

Springer Nature remains neutral with regard to jurisdictional claims in published maps and institutional affiliations.

\section{Author details}

'Animal Bone and Joint Research Laboratory, Department of Veterinary Biosciences and Public Health, Faculty of Veterinary Medicine, Chiang Mai University, Chiang Mai 50100, Thailand. ${ }^{2}$ Department of Food Animal Clinic, Faculty of Veterinary Medicine, Chiang Mai University, Chiang Mai 50200, Thailand. ${ }^{3}$ Department of Biology, Faculty of Science, Chiang Mai University, Chiang Mai 50200, Thailand. ${ }^{4}$ Department of Biochemistry, Faculty of Medicine, Chiang Mai University, Chiang Mai 50200, Thailand. ${ }^{5}$ Excellence Center in Veterinary Bioscience, Chiang Mai University, Chiang Mai 50200, Thailand.

Received: 21 March 2017 Accepted: 28 August 2017

Published online: 06 September 2017

\section{References}

1. Felson DT. Epidemiology of hip and knee osteoarthritis. Epidemiol Rev. 1988;10:1-28.

2. Girodroux M, Dyson S, Murray R. Osteoarthritis of the thoracolumbar synovial intervertebral articulations: clinical and radiographic features in 77 horses with poor performance and back pain. Equine Vet J. 2009;41:130-8

3. Schlueter $A E$, Orth MW. Equine osteoarthritis: a brief review of the disease and its causes. Equine comp Exerc. Physiol. 2004;1:221-31.

4. Slingerland LI, Hazewinkel HAW, Meij BP, Picavet P, Voorhout G. Crosssectional study of the prevalence and clinical features of osteoarthritis in 100 cats. Vet J. 2011;187:304-9.

5. Zhang Y, Jordan JM. Epidemiology of osteoarthritis. Clin Geriatr Med. 2010; 26:355-69.

6. Creamer P. Intra-articular corticosteroid treatment in osteoarthritis. Curr Opin Rheumatol. 1999;11:417-21.

7. Gallelli L, Galasso O, Falcone D, Southworth S, Greco M, Ventura V, et al. The effects of nonsteroidal anti-inflammatory drugs on clinical outcomes, synovial fluid cytokine concentration and signal transduction pathways in knee osteoarthritis. A randomized open label trial. Osteoarthr Cartil. 2013;21:1400-8.

8. Pekarek B, Osher L, Buck S, Bowen M. Intra-articular corticosteroid injections: a critical literature review with up-to-date findings. Foot (Edinb). 2011;21:66-70.

9. Dechant JE, Baxter GM, Frisbie DD, Trotter GW, Mcllwraith CW. Effects of dosage titration of methylprednisolone acetate and triamcinolone acetonide on interleukin-1-conditioned equine articular cartilage explants in vitro. Equine Vet J. 2003;35:444-50.

10. Chrysis D, Zaman F, Chagin AS, Takigawa M, Sävendahl L. Dexamethasone induces apoptosis in proliferative chondrocytes through activation of caspases and suppression of the Akt-phosphatidylinositol 3'-kinase signaling pathway. Endocrinology. 2005;146:1391-7.

11. Chard J, Dieppe P. Update: treatment of osteoarthritis. Arthritis Care Res. 2002;47:686-90.

12. Jonsson H, Olafsdottir S, Sigurdardottir S, Aspelund T, Eiriksdottir G, Sigurdsson $\mathrm{S}$, et al. Incidence and prevalence of total joint replacements due to osteoarthritis in the elderly: risk factors and factors associated with late life prevalence in the AGES-Reykjavik study. BMC Musculoskelet Disord. 2016;17:14.

13. Bannuru RR, Natov NS, Obadan IE, Price LL, Schmid CH, McAlindon T. Therapeutic trajectory of hyaluronic acid versus corticosteroids in the treatment of knee osteoarthritis: a systematic review and meta-analysis. Arthritis Rheum. 2009;61:1704-11.

14. Bellamy N, Campbell J, Robinson V, Gee T, Bourne R, Wells G. Viscosupplementation for the treatment of osteoarthritis of the knee. Cochrane Database Syst Rev. 2006:CD005321.

15. Colen S, van den Bekerom MPJ, Mulier M, Haverkamp D. Hyaluronic acid in the treatment of knee osteoarthritis. BioDrugs. 2012;26:257-68.

16. Medina JM, Thomas A, Denegar CR. Knee osteoarthritis: should your patient opt for hyaluronic acid injection? J Fam Pract. 2006;55:669-75.

17. Miller LE, Block JE. US-approved intra-articular hyaluronic acid injections are safe and effective in patients with knee osteoarthritis: systematic review and meta-analysis of randomized, saline-controlled trials. Clin Med Insights Arthritis Musculoskelet Disord. 2013;6:57-63.

18. Modawal A, Ferrer M, Choi HK, Castle JA. Hyaluronic acid injections relieve knee pain. J Fam Pract. 2005;54:758-67.

19. Rutjes AWS, Jüni $P$, da Costa BR, Trelle $S$, Nüesch E, Reichenbach $S$. Viscosupplementation for osteoarthritis of the knee: a systematic review and meta-analysis. Ann Intern Med. 2012;157:180-91. 
20. Wang C-T, Lin J, Chang C-J, Lin Y-T, Hou S-M. Therapeutic effects of hyaluronic acid on osteoarthritis of the knee: a meta-analysis of randomized controlled trials. J Bone Jt Surg Ser A. 2004;86:538-45.

21. Siengdee P, Euppayo T, Buddhachat K, Chomdej S, Nganvongpanit K. Two fluoroquinolones and their combinations with hyaluronan: comparison of effects on canine chondrocyte culture. J Vet Pharmacol Ther. 2016;39:439-51.

22. Euppayo T, Siengdee P, Buddhachat K, Pradit W, Chomdej S, Ongchai S, et al. Vitro effects of triamcinolone acetonide and in combination with hyaluronan on canine normal and spontaneous osteoarthritis articular cartilage. In Vitro Cell Dev Biol Anim. 2016;52:723-35.

23. Euppayo T, Siengdee P, Buddhachat K, Pradit W, Viriyakhasem N, Chomdej $\mathrm{S}$, et al. Effects of low molecular weight hyaluronan combined with carprofen on canine osteoarthritis articular chondrocytes and cartilage explants in vitro. In Vitro Cell Dev Biol Anim. 2015;51:857-65.

24. Siengdee P, Radeerom T, Kuanoon S, Euppayo T, Pradit W, Chomdej S, et al. Effects of corticosteroids and their combinations with hyaluronanon on the biochemical properties of porcine cartilage explants. BMC Vet Res. 2015;11:298

25. Onur TS, Sitron CS, Dang A. Co-administration of hyaluronic acid with local anaesthetics shows lower cytotoxicity than local anaesthetic treatment alone in bovine articular chondrocytes. Bone Jt Res. 2013;2:270-5.

26. Mihara M, Higo S, Uchiyama Y, Tanabe K, Saito K. Different effects of high molecular weight sodium hyaluronate and NSAID on the progression of the cartilage degeneration in rabbit OA model. Osteoarthr Cartil. 2007;15:543-9.

27. Hashizume M, Mihara M. Desirable effect of combination therapy with high molecular weight hyaluronate and NSAIDs on MMP production. Osteoarthr Cartil. 2009:17:1513-8.

28. Altman R, Brandt K, Hochberg M, Moskowitz R, Bellamy N, Bloch DA, et al. Design and conduct of clinical trials in patients with osteoarthritis: recommendations from a task force of the osteoarthritis research society. Results from a workshop. Osteoarthr Cartil. 1996:4:217-43.

29. Uthman I, Raynauld J, Haraoui B. Intra-articular therapy in osteoarthritis. Postgrad Med J. 2003:79:449-53.

30. de Campos GC, Rezende MU, Pailo AF, Frucchi R, Camargo OP. Adding triamcinolone improves viscosupplementation: a randomized clinical trial. Clin Orthop. 2013;471:613-20.

31. Grecomoro G, Piccione F, Letizia G. Therapeutic synergism between hyaluronic acid and dexamethasone in the intra-articular treatment of osteoarthritis of the knee: a preliminary open study. Curr Med Res Opin. 1992;13:49-55.

32. Lee SC, Rha D-W, Chang WH. Rapid analgesic onset of intra-articular hyaluronic acid with ketorolac in osteoarthritis of the knee. J Back Musculoskelet Rehabil. 2011;24:31-8.

33. Ozturk C, Atamaz F, Hepguler S, Argin M, Arkun R. The safety and efficacy of intraarticular hyaluronan with/without corticosteroid in knee osteoarthritis: 1-year, single-blind, randomized study. Rheumatol Int. 2006;26:314-9.

34. Petrella RJ, Emans PJ, Alleyne J, Dellaert F, Gill DP, Maroney M. Safety and performance of Hydros and Hydros-TA for knee osteoarthritis: a prospective, multicenter, randomized, double-blind feasibility trial. BMC Musculoskelet Disord. 2015;16:57.

35. Dong J, Jiang D, Wang Z, Wu G, Miao L, Huang L. Intra-articular delivery of liposomal celecoxib-hyaluronate combination for the treatment of osteoarthritis in rabbit model. Int J Pharm. 2013:441:285-90.

36. Karakurum G, Karakok M, Tarakcioglu M, Kocer NE, Kocabas R, Bagci C. Comparative effect of intra-articular administration of hyaluronan and/or cortisone with evaluation of malondialdehyde on degenerative osteoarthritis of the rabbit's knee. Tohoku J Exp Med. 2003;199:127-34.

37. Zhang Z, Wei X, Gao J, Zhao Y, Zhao Y, Guo L, et al. Intra-articular injection of cross-linked hyaluronic acid-dexamethasone hydrogel attenuates osteoarthritis: an experimental study in a rat model of osteoarthritis. Int J Mol Sci. 2016;17:411.

38. Doyle AJ, Stewart AA, Constable PD, Eurell JAC, Freeman DE, Griffon DJ. Effects of sodium hyaluronate and methylprednisolone acetate on proteoglycan synthesis in equine articular cartilage explants. Am J Vet Res. 2005;66:48-53.

39. Schaefer EC, Stewart AA, Durgam SS, Byron CR, Stewart MC. Effects of sodium hyaluronate and triamcinolone acetonide on glucosaminoglycan metabolism in equine articular chondrocytes treated with interleukin-1. Am J Vet Res. 2009;70:1494-501.

40. Yates AC, Stewart AA, Byron CR, Pondenis HC, Kaufmann KM, Constable PD. Effects of sodium hyaluronate and methylprednisolone acetate on proteoglycan metabolism in equine articular chondrocytes treated with interleukin-1. Am J Vet Res. 2006;67:1980-6.
41. Caborn D, Rush J, Lanzer W, Parenti D, Murray C. Synvisc 901 study group. A randomized, single-blind comparison of the efficacy and tolerability of hylan G-F 20 and triamcinolone hexacetonide in patients with osteoarthritis of the knee. J Rheumatol. 2004;31:333-43.

42. Price DD, Bush FM, Long S, Harkins SWA. Comparison of pain measurement characteristics of mechanical visual analogue and simple numerical rating scales. Pain. 1994;56:217-26.

43. Averbuch M, Katzper M. Assessment of visual analog versus categorical scale for measurement of osteoarthritis pain. J Clin Pharmacol. 2004;44: 368-72.

44. Bellamy N, Buchanan WW, Goldsmith CH, Campbell J, Stitt LW. Validation study of WOMAC: a health status instrument for measuring clinically important patient relevant outcomes to antirheumatic drug therapy in patients with osteoarthritis of the hip or knee. J Rheumatol. 1988;15:1833-40.

45. Salaffi F, Leardini G, Canesi B, Mannoni A, Fioravanti A, Caporali R, et al. Reliability and validity of the western Ontario and McMaster universities (WOMAC) osteoarthritis index in Italian patients with osteoarthritis of the knee. Osteoarthr Cartil. 2003;11:551-60.

46. Söderman P, Malchau H. Validity and reliability of Swedish WOMAC osteoarthritis index: a self-administered disease-specific questionnaire (WOMAC) versus generic instruments (SF-36 and NHP). Acta Orthop Scand. 2000;71:39-46.

47. Tüzün EH, Eker L, Aytar A, Daşkapan A, Bayramoğlu M. Acceptability, reliability, validity and responsiveness of the Turkish version of WOMAC osteoarthritis index. Osteoarthr Cartil. 2005;13:28-33.

48. Bruyère O, Cooper C, Pelletier J-P, Branco J, Brandi ML, Guillemin F, et al. An algorithm recommendation for the management of knee osteoarthritis in Europe and internationally: a report from a task force of the European Society for Clinical and Economic Aspects of osteoporosis and osteoarthritis (ESCEO). Semin Arthritis Rheum. 2014:44:253-63.

49. McAlindon TE, Bannuru RR, Sullivan MC, Arden NK, Berenbaum F, BiermaZeinstra SM, et al. OARSI guidelines for the non-surgical management of knee osteoarthritis. Osteoarthr Cartil. 2014;22:363-88.

50. Chen AL, Desai P, Adler EM, Di Cesare PE. Granulomatous inflammation after Hylan G-F 20 viscosupplementation of the knee. J Bone Jt Surg Am. 2002; 84:1142-7.

51. Allen E, Krohn K. Adverse reaction to Hylan GF-20. J Rheumatol. 2000;27: 1572.

52. Moskowitz RW. Osteoarthritis: diagnosis and medical/surgical management. Philadelphia: Lippincott Williams \& Wilkins; 2007.

53. Bruyère $\mathrm{O}$, Cooper C, Pelletier JP, Maheu E, Rannou F, Branco J, et al. A consensus statement on the European Society for Clinical and Economic Aspects of osteoporosis and osteoarthritis (ESCEO) algorithm for the management of knee osteoarthritis-from evidence-based medicine to the real-life setting. Semin Arthritis Rheum. 2016;45:S3-11.

54. Pyne D, loannou Y, Mootoo R, Bhanji A. Intra-articular steroids in knee osteoarthritis: a comparative study of triamcinolone hexacetonide and methylprednisolone acetate. Clin Rheumatol. 2004;23:116-20.

55. Eberhard BA, Sison MC, Gottlieb BS, llowite NT. Comparison of the intraarticular effectiveness of triamcinolone hexacetonide and triamcinolone acetonide in treatment of juvenile rheumatoid arthritis. J Rheumatol. 2004; 31:2507-12

56. Stein A, Yassouridis A, Szopko C, Helmke K, Stein C. Intraarticular morphine versus dexamethasone in chronic arthritis. Pain. 1999;83:525-32.

57. Calmet J, Esteve C, Boada S, Giné J. Analgesic effect of intra-articular ketorolac in knee arthroscopy: comparison of morphine and bupivacaine. Knee Surg Sports Traumatol Arthrosc. 2004;12:552-5.

58. Ng HP, Nordström U, Axelsson K, Perniola AD, Gustav E, Ryttberg L, et al. Efficacy of intra-articular bupivacaine, ropivacaine, or a combination of ropivacaine, morphine, and ketorolac on postoperative pain relief after ambulatory arthroscopic knee surgery: a randomized double-blind study. Reg Anesth Pain Med. 2006:31:26-33.

59. Jiang D, Zou J, Huang L, Shi Q, Zhu X, Wang G, et al. Efficacy of intraarticular injection of celecoxib in a rabbit model of osteoarthritis. Int J Mol Sci. 2010;11:4106-13.

60. Pelletier JP, Lajeunesse D, Jovanovic DV, Lascau-Coman V, Jolicoeur FC, Hilal $\mathrm{G}$, et al. Carprofen simultaneously reduces progression of morphological changes in cartilage and subchondral bone in experimental dog osteoarthritis. J Rheumatol. 2000;27:2893-902.

61. Huang K, Aggrecanase WLD. Aggrecan degradation in osteoarthritis: a review. J Int Med Res. 2008;36:1149-60. 
62. Poole AR, Kobayashi M, Yasuda T, Laverty S, Mwale F, Kojima T, et al. Type II collagen degradation and its regulation in articular cartilage in osteoarthritis. Ann Rheum Dis. 2002;61:ii78-81.

63. Maheu E, Rannou F, Reginster JY. Efficacy and safety of hyaluronic acid in the management of osteoarthritis: evidence from real-life setting trials and surveys. Semin Arthritis Rheum. 2016:45:S28-33.

64. Egger M, Jüni P, Bartlett C, Holenstein F, Sterne J. How important are comprehensive literature searches and the assessment of trial quality in systematic reviews? Empirical study Health Technol Assess (Winch Engl). 2003;7:1-76.

65. Kellgren JH, Lawrence JS. Radiological assessment of osteo-arthrosis. Ann Rheum Dis. 1957;16:494-502.

66. Kellgren JH, Lawrence JS. Radiological assessment of rheumatoid arthritis. Ann Rheum Dis. 1957;16:485-93.

67. Karakok M, Ugras S, Tosun N, Fuat A, Aydin A. Effect of intra-articular administration of hyaluronan and cortisone on secondary osteoarthritis of the infected rabbit's knee. Tohoku J Exp Med. 2001;195:35-42.

68. Kikuchi T, Yamada H, Shimmei M. Effect of high molecular weight hyaluronan on cartilage degeneration in a rabbit model of osteoarthritis. Osteoarthr Cartil. 1996:4:99-110

69. Gerwin N, Bendele AM, Glasson S, Carlson CS. The OARSI histopathology initiative - recommendations for histological assessments of osteoarthritis in the rat. Osteoarthr Cartil 2010;18(Suppl 3):S24-24.

\section{Submit your next manuscript to BioMed Central and we will help you at every step:}

- We accept pre-submission inquiries

- Our selector tool helps you to find the most relevant journal

- We provide round the clock customer support

- Convenient online submission

- Thorough peer review

- Inclusion in PubMed and all major indexing services

- Maximum visibility for your research

Submit your manuscript at www.biomedcentral.com/submit 